\title{
Spectroscopie du plasma induit par laser pour l'analyse de matière organique
}

\author{
Jin YU et Vincent MOTTO-ROS \\ Institut Lumière Matière - Université de Lyon \\ jin.yu@univ-lyon1.fr
}

La spectroscopie d'émission du plasma induit par laser conduit à la technique LIBS, acronyme qui signifie laserinduced breakdown spectroscopy en anglais. Dans cette technique, un petit plasma est généré en focalisant une impulsion laser sur un échantillon d'intérêt, qu'il soit solide, liquide ou gazeux. L'analyse spectrale de la lumière émise par le plasma permet d'identifier et de quantifier les éléments contenus initialement dans le volume ablaté de l'échantillon. La polyvalence, la facilité de mise en œuvre, la rapidité de réponse, la sensibilité et la possibilité de réaliser des analyses à distance font de la LIBS une technique à très fort potentiel applicatif, ceci particulièrement dans les domaines liés à la protection de l'environnement. Dans cet article, nous faisons le point sur l'application de cette technique à la matière organique, application suscitée notamment par des besoins en détection de polluants tels que les métaux lourds dans l'environnement et par le recyclage de déchets plastiques.

\section{Du laser à LIBS, 50 ans d'une technique analytique en constante évolution}

Deux ans après l'invention du laser, en 1962, deux physiciens américains, F. Brech et $\mathrm{L}$. Cross, découvrirent le plasma induit par laser lorsqu'ils focalisèrent une impulsion sur la surface d'une cible. Celui-ci fut observé sous la forme d'une micro-émission optique. L'analyse spectroscopique de l'émission effectuée un an plus tard par deux physiciennes françaises, J. DebrasGuédon et N. Liodec, révéla un riche spectre d'émission d'éléments (ions et atomes) aussi bien que de molécules (et de radicaux). Les énergies d'excitation des émissions vont de quelques eV à une dizaine d'eV, attestant une température du plasma dépassant une dizaine de milliers de kelvin.

Ces découvertes fondamentales restèrent cependant longtemps une curiosité de laboratoire jusqu'aux années 80 où les premières applications ont été démontrées avec les travaux de D.A. Cremers et L.J. Radziemski à Los Almos National Laboratory pour la détection d'aérosols dans l'air contenant des éléments métalliques (béryllium) ou non métalliques (chlore ou fluore). Les progrès technolo- giques des lasers, des spectromètres et des détecteurs (CCD et ICCD) ont alors facilité le développement d'instruments LIBS qui sont apparus au milieu des années 80 . Aujourd'hui la technique LIBS est amenée à répondre aux besoins de détection et d'analyse dans des domaines aussi variés que l'environnement, I'agroalimentaire, la protection du patrimoine, I'analyse biomédicale, la défense et la sécurité nationale, sans oublier l'exploration spatiale. Le rover Curiosity envoyé par la NASA sur Mars se déplace actuellement sur la planète avec un instrument LIBS à la recherche des conditions favorables à I'apparition de la vie. Des spectres LIBS du sol martien sont ainsi transmis régulièrement sur Terre pour être analysés.

50 ans après son apparition et face aux développements florissants des applications, la technique LIBS est en passe de devenir une technique analytique de choix qui s'impose grâce aux avantages qui lui sont propres : la polyvalence pour analyser directement, sans préparation élaborée, des échantillons sous n'importe quelle forme (solide, liquide ou gazeuse) ; une rapidité de réponse qui permet de fournir un résultat d'analyse en une fraction de seconde favorable pour des ana- lyses en ligne ; une sensibilité de l'ordre du ppm en masse pour la plupart des éléments du tableau périodique ; une capacité de mesure sans contact pour des analyses à distance ; et une résolution spatiale micrométrique pour de la microanalyse ou la cartographie élémentaire de surface. La maturation de la technique LIBS ne peut cependant se faire sans obstacle. Son tendon d'Achille est lié à la complexité des processus impliqués dans la formation du plasma induit par laser (avec typiquement des impulsions nanosecondes). Aujourd'hui, la LIBS souffre encore, par rapport aux techniques analytiques classiques telles que I'ICP-AES ou I'ICP-MS, des insuffisances en termes de précision, de répétabilité et de sensibilité (notamment pour des éléments à niveaux excités énergétiques). Le contraste est saisissant entre la simplicité de mise en œuvre de la technique, qui favorise les applications, et la complexité des processus physiques impliqués, qui nécessite une étude fondamentale poussée pour justement soutenir le développement de ces applications. Après avoir connu une longue période essentiellement consacrée au développement applicatif, la communauté internationale LIBS considère aujourd'hui 
qu'un retour à l'étude fondamentale du plasma induit par laser est une nécessité pour la maturation de la technique.

\section{Besoins et défis pour \\ I'application LIBS à la matière organique}

Restée longtemps appliquée à l'analyse de matière inorganique, en particulier des métaux et des minéraux, la LIBS connaît récemment un fort développement pour son application à la matière organique ou biologique. Nous pouvons citer la détection et l'identification d'explosifs, l'analyse des métaux toxiques dans les produits agroalimentaires, ou la discrimination et l'identification de déchets plastiques. Son application à la matière organique amène de nouvelles difficultés. Premièrement, l'ablation d'une matrice organique met en jeu des processus bien plus complexes que dans le cas d'un métal, ce qui conduit à des effets de matrice plus importants, c'est-à-dire une dépendance accrue de l'émission du plasma aux propriétés physico-chimiques de l'échantillon analysé. Un tel effet rend difficile la quantification par la procédure classique d'étalonnage entre l'intensité d'émission d'une espèce dans le plasma et sa concentration dans l'échantillon. L'ablation stœchiométrique (composition élémentaire du plasma identique à celle de l'échantillon) est également plus difficile à atteindre. Une deuxième difficulté provient du fait que la plupart des matériaux organiques sont assez similaires du point de vue élémentaire, car les éléments organiques tels que $\mathrm{C}, \mathrm{H}, \mathrm{O}$ et $\mathrm{N}$ y sont omniprésents. La LIBS est essentiellement une technique d'analyse élémentaire, due à la dominance des émissions des espèces élémentaires (atomes ou ions) dans le plasma, ce qui rend son application à la matière organique plus difficile.

\section{Montage expérimental utilisé}

À l'Institut Lumière Matière à l'université Lyon 1, nous développons des applications LIBS à la matière organique depuis 2005. Un montage standard, typiquement utilisé, est illustré à la figure 1. Un laser $\mathrm{Nd}$ :YAG permet l'ablation avec des impulsions ultraviolettes ( 266 ou $355 \mathrm{~nm}$ ) de quelques nanosecondes de durée. L'énergie par impulsion est typiquement de 10 à $50 \mathrm{~mJ}$. Un obturateur mécanique est utilisé pour contrôler le tir laser sur un échantillon. Les impulsions laser sont focalisées sur la surface de celui-ci par une lentille ayant une distance focale de $5 \mathrm{~cm}$. L'émission du plasma créé est collectée par un système optique à deux lentilles et est couplée à un spectromètre à échelle via une fibre optique. Le spectromètre est équipé d'une caméra ICCD qui enregistre le spectre d'une manière synchronisée par rapport à l'impact de l'impulsion laser sur l'échantillon. Le délai de détection de l'émission par I'ICCD est typiquement de

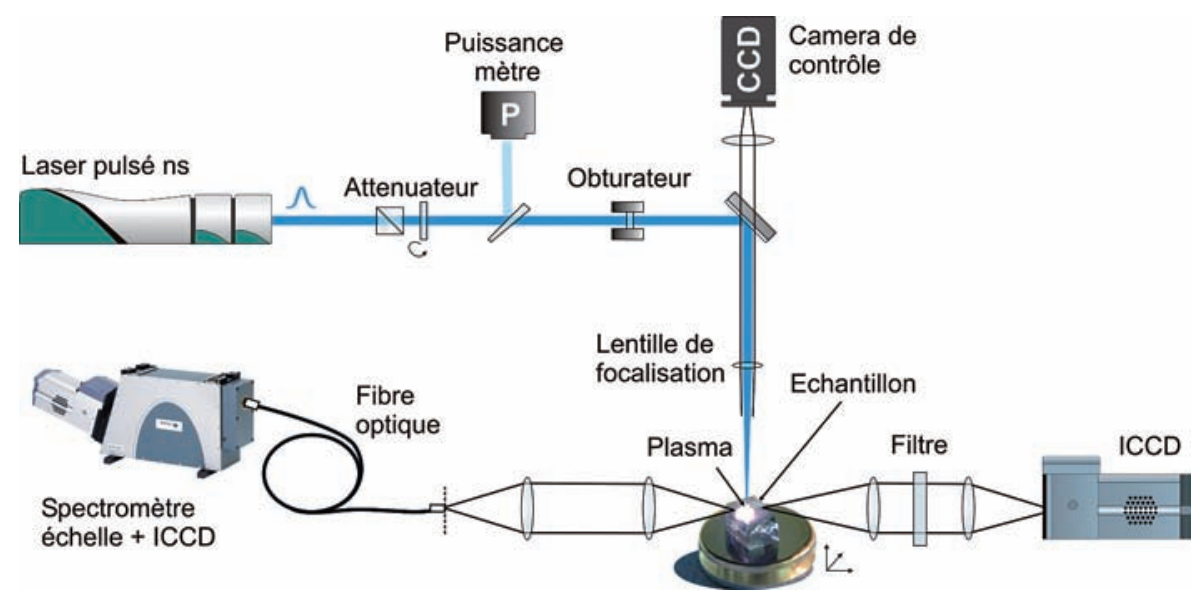

Figure 1. Présentation schématique du montage expérimental. 

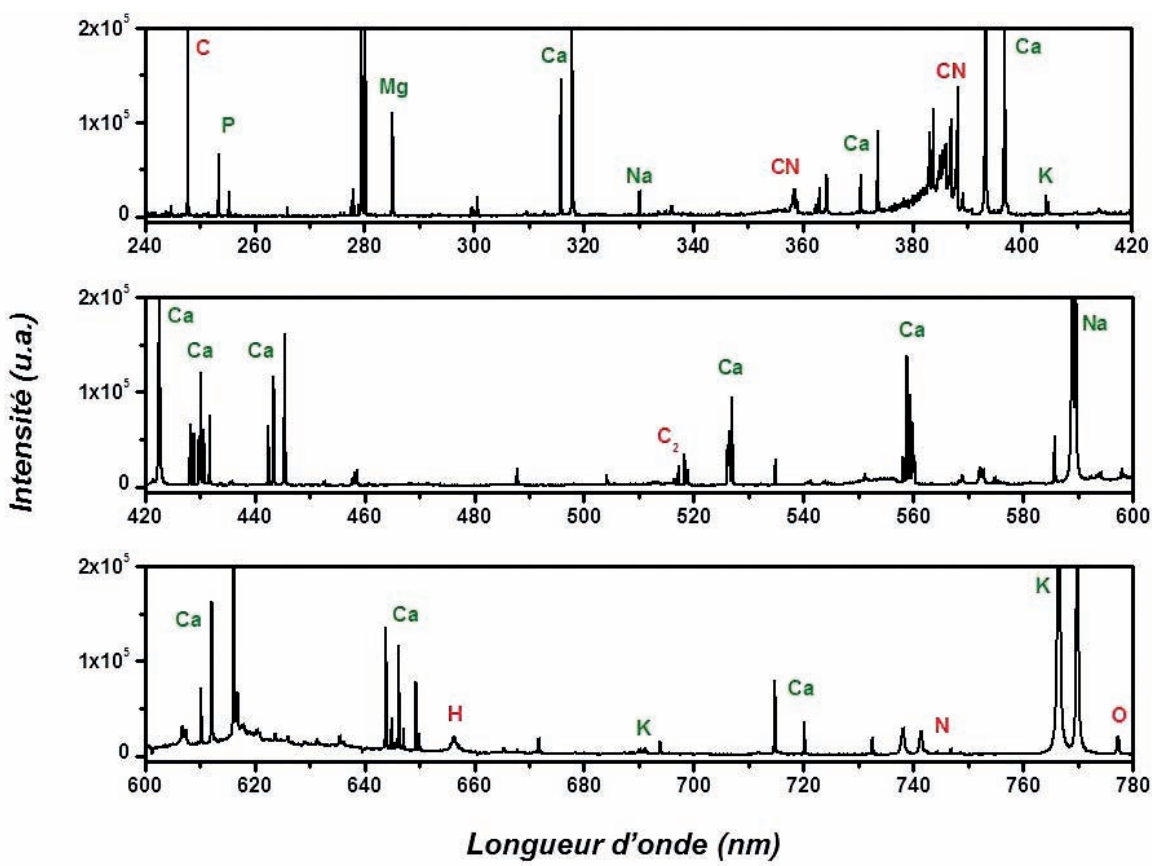

Figure 2. Spectre LIBS typique d'un échantillon de lait en poudre compressé en pastille. Dans ce spectre, nous pouvons identifier des raies d'émission d'éléments inorganiques (notées en vert), et celles d'éléments organiques (notées en rouge). Des éléments organiques peuvent apparaître aussi en molécules diatomiques telles que $\mathrm{CN}$ et $\mathrm{C}_{2}$.

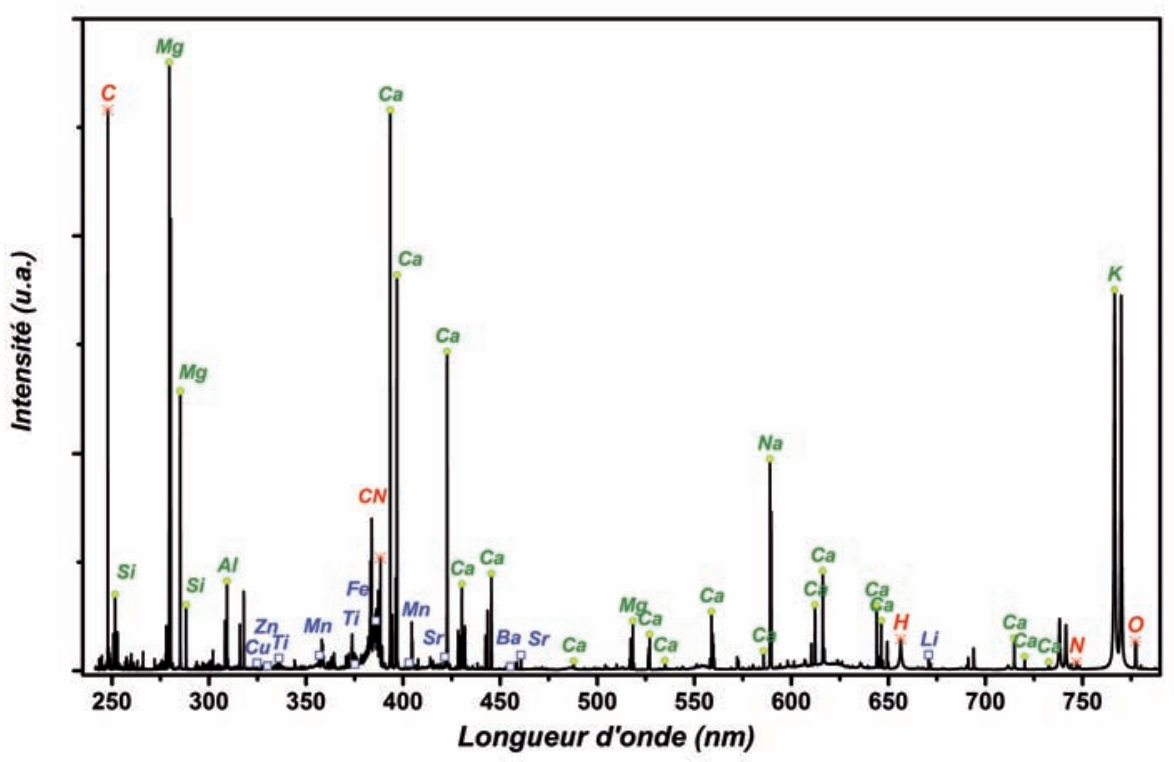

Figure 3. Spectre LIBS de la peau d'une pomme de terre montrant la présence des éléments organiques: $\mathrm{C}, \mathrm{H}, \mathrm{N}, \mathrm{O}$ (notés en rouge) ; des majeurs : $\mathrm{Mg}, \mathrm{Ca}, \mathrm{Si}, \mathrm{Al}, \mathrm{K}, \mathrm{Na}$ (notés en vert); et des traces : $\mathrm{Fe}, \mathrm{Ti}$, $\mathrm{Mn}, \mathrm{Sr}, \mathrm{Ba}, \mathrm{Li}, \mathrm{Rb}, \mathrm{Cu}, \mathrm{Zn}, \mathrm{Cr}$ (notés en bleu). Une vingtaine d'éléments sont donc détectés par LIBS dans la peau d'une pomme de terre.

500 ns après l'initiation du plasma afin d'éviter l'émission du continuum à temps court. Une variante du montage permet d'enregistrer l'image du plasma directe- ment par la caméra en positionnant la matrice photosensible dans le plan image du système de collecte. Dans ce cas-là, un filtre passe-bande est utilisé pour sélec- tionner une raie d'émission de l'espèce étudiée. Il est possible d'enregistrer le spectre ou l'image spectrale du plasma avec une résolution temporelle de l'ordre de 5 ns avec un contrôle sur l'activation de l'intensificateur de la caméra. Un spectre LIBS typique d'un échantillon organique (lait en poudre) est illustré à la figure 2. Nous pouvons identifier sur ce spectre des raies d'émission spécifiques aux éléments inorganiques (notés en vert) et aux éléments organiques (notés en rouge). Les éléments organiques peuvent également apparaître sous la forme de molécules diatomiques telles que $\mathrm{CN}$ et $\mathrm{C}_{2}$. Dans notre équipe, nous développons I'application LIBS à l'analyse de matière organique sur deux axes de recherches: d'une part, la détection et la quantification d'éléments-traces métalliques ; d'autre part la discrimination et l'identification des composés organiques par des méthodes chimiométriques ou par l'étude du comportement des fragments moléculaires dans le plasma.

\section{Analyse des éléments-traces métalliques dans une matrice organique}

\section{Le cas de la pomme de terre [1]}

L'analyse de pommes de terre représente un cas d'école pour l'analyse de produits agroalimentaires frais par LIBS. Mis à part sa forme, qui facilite l'ablation directe sur la peau, le fait qu'elle soit en contact direct avec la terre de production, permet de détecter une éventuelle pollution de celle-ci. La figure 3 présente un spectre LIBS typique de la peau d'une pomme de terre. Le spectre montre la présence d'éléments organiques $(C, H, N$, O), de majeurs ( $\mathrm{Mg}, \mathrm{Ca}, \mathrm{Si}, \mathrm{Al}, \mathrm{K}, \mathrm{Na}$ ) et de traces (Fe, Ti, Mn, Sr, Ba, Li, Rb, Cu, Zn, Cr). Une vingtaine d'éléments sont donc détectés dans le cas présent, ce qui montre la sensibilité de la technique. La quantification des éléments reste cependant inaccessible tant que l'étalonnage ne peut être réalisé. II est nécessaire pour cela d'établir une relation entre l'intensité d'émission d'un élément et sa concentration en utilisant des échantillons de 
référence de matrice similaire au produit analysé. Pour un légume frais, il est cependant quasiment impossible d'obtenir de telles références. Une alternative est d'utiliser la procédure de calibration-free LIBS (CF-LIBS) (voir encadré), qui consiste à calculer les concentrations des éléments détectés directement à partir du spectre LIBS en supposant que le plasma est en équilibre thermodynamique local (ETL). Pour le plasma induit par laser, l'ETL ne représente, dans le meilleur des cas, qu'une approximation de son état thermodynamique réel. Les résultats de calcul par CF-LIBS sont donc assujettis à d'importantes incertitudes et doivent être validés par une technique analytique indépendante.

\section{Analyse de poudres de lait [2]}

Afin de valider la procédure CF-LIBS pour l'analyse de matière organique, nous avons comparé des résultats d'analyse obtenus avec LIBS à ceux obtenus en ICP-AES pour les mêmes échantillons. Des poudres de lait pour nourrissons ont été choisies pour cette étude, car elles peuvent être facilement préparées aussi bien en pastille pour l'analyse LIBS qu'en solution pour l'analyse ICP-AES. De plus, ces poudres sont fournies avec la concentration nominale des minéraux présents, ce qui permet de les utiliser comme des échantillons de référence. Les concentrations de magnésium et de potassium déterminées par CF-LIBS sont présentées à la figure 4 ainsi que les concentrations nominales et celles mesurées par ICP-AES. À partir de ces résultats, les incertitudes relatives de mesure LIBS, moyennées sur l'ensemble des échantillons, sont de $24 \%$ pour Mg et $16 \%$ pour K. Ces valeurs restent encore bien supérieures à celles des mesures ICP-AES qui donnent 4,1\% et $4,4 \%$ respectivement. Les déviations des concentrations mesurées par rapport aux valeurs nominales sont de 17,6\% $(\mathrm{Mg})$ et $12,1 \%(\mathrm{~K})$ pour la détermination CF-LIBS, alors qu'elles sont de $6,4 \%$ et $4,4 \%$ pour la mesure ICP-AES. Ces résultats montrent une performance relativement satisfaisante de la procédure CF-LIBS pour I'analyse directe de matière organique (sans préparation préalable de solution), bien qu'elle reste encore clairement moins précise et moins exacte que I'ICP-AES.

\section{Plasma en équilibre thermodynamique local (ETL) et calibration-free LIBS}

Le plasma, le quatrième état de la matière, correspond à un gaz ionisé. L'état thermodynamique d'un plasma peut être décrit par les lois de distribution qui sont la distribution de Maxwell pour les vitesses des particules, la distribution de Boltzmann pour les populations parmi les niveaux internes d'une espèce (neutre ou ion d'un élément), la distribution de Saha pour les populations parmi les différents états d'ionisation d'un même élément, et la distribution de Planck pour l'énergie de photons.

L'équilibre thermodynamique complet (ETC) d'un plasma correspond à un état où les températures caractéristiques des 4 lois de distribution sont identiques. Le plasma correspond alors dans ce cas-là à un corps noir. Pour un plasma induit par laser, I'ETC est en général impossible du fait que l'énergie contenue dans le rayonnement est négligeable devant celles contenues dans des particules matérielles, comme les électrons, atomes, ions et molécules. À condition que des collisions dans le plasma, notamment celles dues à des électrons, soient suffisamment rapides pour qu'elles soient dominantes dans la détermination des populations parmi les niveaux internes $d^{\prime}$ 'une espèce et dans les différents états d'ionisation d'un même élément, le plasma peut atteindre un état d'équilibre partiel appelé équilibre thermodynamique local (ETL). Un état de l'ETL est défini par le fait que les lois de distribution de Maxwell, de Boltzmann et de Saha sont caractérisées par une température unique. Pour un plasma à l'ETL, si l'on arrive à déterminer la densité électronique par l'élargissement Stark et la température par les diagrammes de Boltzmann ou de Saha-Boltzmann, l'utilisation des lois de distribution de Boltzmann et de Saha nous permet de relier l'intensité d'émission d'une raie à la concen tration relative de l'élément correspondant. Toutefois, les paramètres spectroscopiques relatifs à cette raie doivent être connus avec une base de données spectroscopiques, par exemple celle du NIST. Une condition de normalisation, qu'elle soit la prise en compte de tous les éléments dans le plasma ou encore la neutralité de celvi-ci, permet finalement de calculer la concentration absolue de l'élément. Cette procédure correspond à ce qu'on appelle dans la littérature la calibration-free LIBS ou la CF-LIBS.

\section{TIOOS-HP Lasers Accordables}

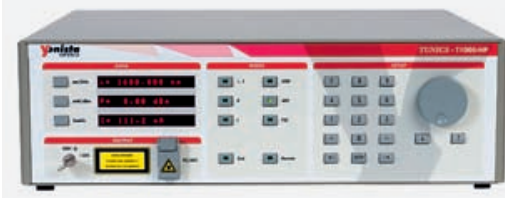

Caractéristiques clés :

Puissance $+8 \mathrm{dBm}$

SSSER $>90 \mathrm{~dB}$

Précision : $\pm 20 \mathrm{pm}$

Balayage sans sauts de mode

Fibre : SMF ou PMF

Modèles disponibles:

$$
\begin{array}{r}
\text { /O : } 1240-1380 \mathrm{~nm} \\
/ \mathrm{ES}: 1340-1520 \mathrm{~nm} \\
/ \mathrm{SCL}: 1440-1640 \mathrm{~nm} \\
/ \mathrm{CL}: 1500-1630 \mathrm{~nm} \\
/ C L U: 1500-1700 \mathrm{~nm}
\end{array}
$$

\section{Technologie T100}

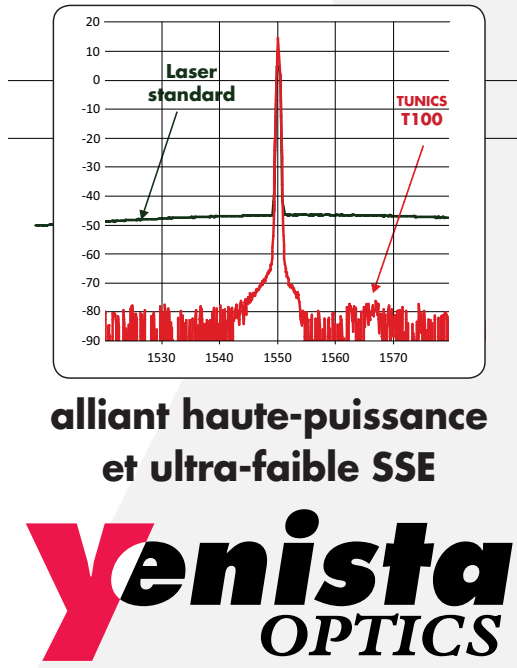

$$
\begin{gathered}
\text { Tél. : +33 (0)2964837 } 16 \\
\text { sales-emea@yenista.com } \\
\text { www.yenista.com }
\end{gathered}
$$



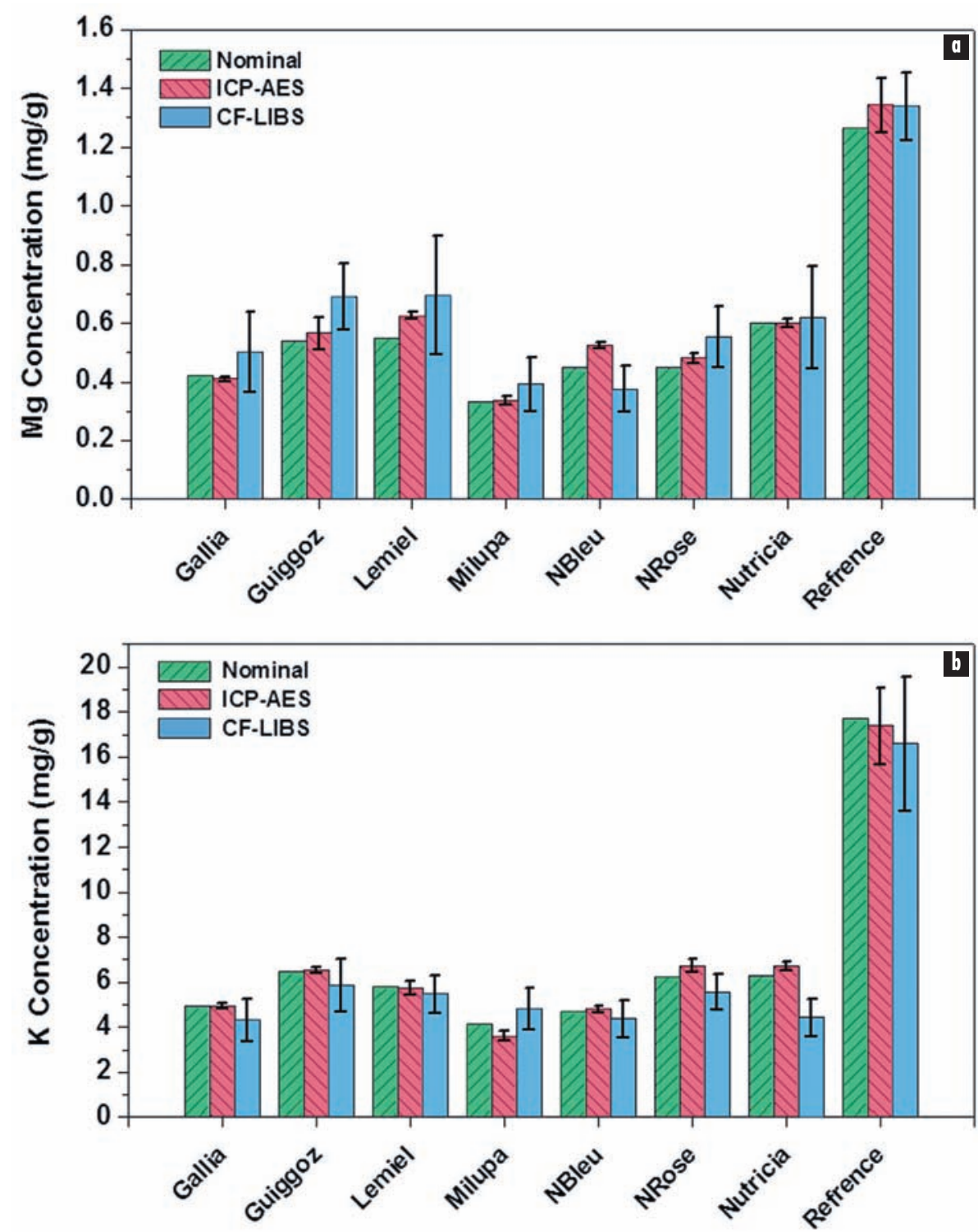

Figure 4. Concentrations de magnésium (a) et de potassium (b) déterminées par CF-LIBS dans des poudres de lait pour nourrissons comparées avec les valeurs nominales et celles mesurées par ICP-AES pour les même échantillons.

\section{Discrimination et identification de matrices organiques}

Le tri automatisé des déchets plastiques repose sur l'identification de différentes familles de matrices organiques. Cette application représente aujourd'hui un enjeu majeur dans le cadre du développement durable et face à la pénurie annoncée des ressources pétrolières. La LIBS semble très prometteuse pour cette application car en plus de pouvoir identifier les différentes familles de plastiques, elle est capable de quantifier les additifs, souvent toxiques, ajoutés lors de la fabri- figure 5. Après l'acquisition des spectres, une partie de ceux-ci est utilisée pour l'apprentissage du réseau de neurones. Une fois le réseau entrainé, il est capable de répondre instantanément et sans supervision pour un spectre inconnu en lui associant soit l'échantillon le plus proche de sa base d'apprentissage, soit une nonidentification. Les résultats d'identification pour des spectres pris avec les 8 échantillons étudiés sont montrés à la figure 5. Ces résultats ont été obtenus avec des spectres uniques sans accumulation. Dans cette configuration, le réseau est capable de traiter 10 spectres par seconde. Nous voyons que pour des spectres uniques, un taux d'identification correct est observé pour 7 échantillons parmi les 8 testés, à l'exception du POM. Nous pouvons par ailleurs signaler que les échantillons sont tous bien identifiés avec des spectres moyennés à partir de 5 accumulations.

\section{Évolutions spatiale et temporelle des fragments moléculaires [4]}

Cette approche consiste à extraire des informations supplémentaires pour l'identification d'un polymère en observant l'évolution spatio-temporelle des fragments moléculaires dans le plasma. Nous avons démontré précédemment que le comportement de l'évolution temporelle du radical $C N$ est corrélé avec la présence ou non de la liaison CN native dans la matrice organique initiale [5]. L'utilisation de la technique d'imagerie spectrale différentielle [6] apporte l'information supplémentaire concernant la distribution spatiale des molécules dans le plasma. Afin d'étudier la corrélation entre la localisation des fragments moléculaires dans le plasma et la structure moléculaire de la matrice, nous avons étudié 4 polymères de structure spécifique : PA6-6 (Nylon) comportant des liaisons $\mathrm{CN}$ et $\mathrm{CC}$ natives, $P S$ et $P E$ ayant respectivement des liaisons doubles $C=C$ et simple $C-C$, et POM sans liaison $\mathrm{CN}$ et $\mathrm{CC}$ native. La figure 6 montre les images des coefficients d'émission des 3 espèces étudiées dans le plasma, $C N, C_{2}$ et $\mathrm{N}$, à un délai de $600 \mathrm{~ns}$. Ces images ont été obtenues après inversion d'Abel des images spectrales brutes. Elles mettent en évidence la corrélation entre les distribu- 


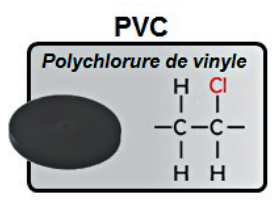

PP

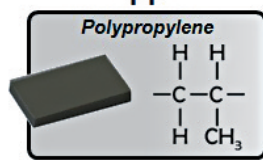

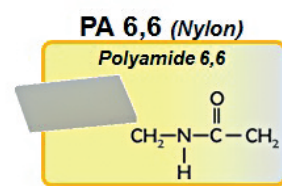

POM
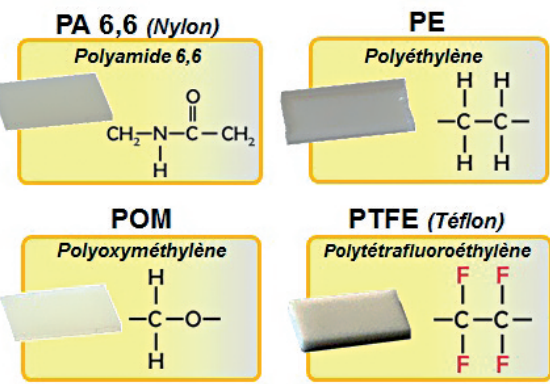

PTFE (Téflon)
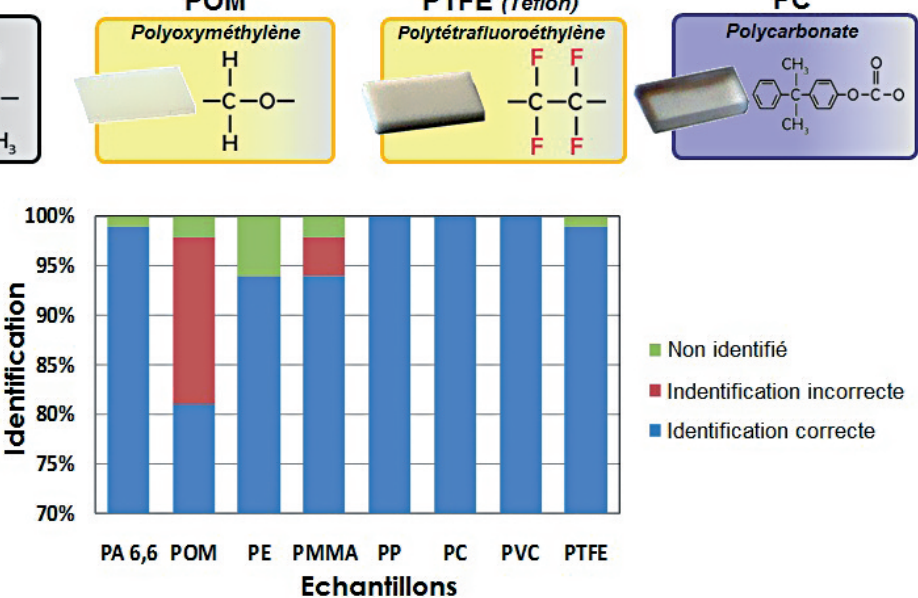

n Non identifié

- Indentification incorrecte

- Identification correcte

Figure 5. Échantillons étudiés et résultats d'identification avec des spectres uniques sans accumulation avec un réseau de neurones artificiels.


Figure 6. Images en coefficient d'émission des 3 espèces dans le plasma, $\mathrm{CN}, \mathrm{C}_{2}$ et $\mathrm{N}$, pour les 4 échantillons de polymères purs (a) PA6-6, (b) $P S$, (c) PE, et (d) POM, prises à un délai de 600 ns. Les correspondances des couleurs utilisées sont: le rouge pour le radical $\mathrm{CN}$, le vert pour la molécule $C_{2}$, et le bleu pour l'élément $N$. Le trait en pointillé indique la surface de l'échantillon.

tions des molécules (proportionnelles aux coefficients d'émission) dans le plasma et la structure moléculaire du polymère ablaté. Ainsi le POM se distingue en premier lieu par sa très faible émission $(\approx 100$ fois plus faible) de $C_{2}$ conformément à l'absence de liaison CC dans sa matrice.
Le PA se différencie ensuite du groupe de PS et PE par la présence du radical $\mathrm{CN}$ au centre du plasma, due à l'existence de liaisons CN natives. Enfin, la discrimination entre le PS et le PE se fait avec la différence en distribution de $C_{2}$ dans le plasma. L'existence de double liaison $C=C$ dans PS conduit donc à une distribution spécifique de molécules $C_{2}$ dans le plasma. Un algorithme sera ainsi capable de discriminer et d'identifier des matrices de polymères différentes en partant des distributions des molécules observées.

\section{Conclusion}

Nous avons présenté, dans leurs grandes lignes, nos résultats obtenus dans l'application LIBS à I'analyse de matière organique. Cette application a un très fort potentiel mais présente encore des défis à relever.

L'un des axes explorés concerne la détection et la quantification d'éléments métalliques présents dans une matrice organique sous forme d'éléments mineurs ou de trace. Nous avons démontré la sensibilité de la LIBS pour la détection d'un grand nombre d'éléments dans une matrice organique typique et de grand inté- rêt environnemental telle que la peau d'une pomme de terre. La quantification des éléments détectés reste cependant difficile en l'absence d'échantillons de référence. La procédure CF-LIBS apparaît alors comme un recours indispensable et nous avons montré qu'elle était capable de fournir des résultats d'assez bonne qualité. Néanmoins, la performance de la CF-LIBS reste à améliorer d'une manière significative pour arriver aux performances des techniques analytiques établies. Une meilleure compréhension et une modélisation détaillée du plasma induit par laser constituent ainsi une solution pertinente.

Un deuxième axe exploré vise à trouver des méthodes efficaces et fiables pour discriminer et identifier différentes familles de composés organiques. L'utilisation d'un réseau de neurones artificiels a démontré son efficacité. Sa fiabilité reste cependant à valider avec un nombre significativement plus élevé d'échantillons. Une détection des émissions des fragments moléculaires ou radicalaires du plasma, résolue temporellement et spatialement, peut fournir une signature spécifique d'un composé organique selon sa structure moléculaire. Ce procédé démontré avec des polymères purs reste à valider avec des échantillons de plastique contenant des impuretés (charges et aditifs). Finalement, les résultats présentés dans cet article donnent un aperçu encourageant de I'application de la technique LIBS à la matière organique. Avec son fort potentiel d'application, la LIBS comptera forcément dans l'avenir comme une technique analytique de choix pour des domaines liés à l'environnement.

\section{Références}

[1] Juvé et al., Spectrochim. Acta Part B, 63, 1047 (2008)

[2] Lei et al., Anal. Bioanal. Chem., 400, 3303 (2011)

[3] Boveri et al., Appl. Spectrosc., 65, 307 (2011)

[4] Grégoire et al., Spectrochim. Acta Part B, 74-75, 31 (2012)

[5] Baudelet et al., Appl. Phys. Lett., 88, 063901 (2006)

[6] Motto-Ros et al., Spectrochim. Acta Part B, 74-75, 11 (2012) 